\title{
CONTRIBUIÇÕES DA PSICOLOGIA COMPORTAMENTAL SOBRE HÁBITOS DE ESTUDO EM CONTEXTO UNIVERSITÁRIO
}

\section{CONTRIBUTIONS OF BEHAVIORAL PSYCHOLOGY ON STUDY HABITS IN UNIVERSITY CONTEXT}

\author{
Isnara Gomes Teixeira \\ Acadêmica de Psicologia, Centro Universitário Doctum de Teófilo Otoni, Brasil \\ E-Mail: isnaragomes@gmail.com \\ João Carlos Muniz Martinelli \\ Professor Ms. Psicologia Clínica \\ E- Mail: joaocmmartinelli@gmail.com \\ Lucio Onofri \\ Professor de Técnicas de Pesquisa \\ E-mail: lucioonofri@gmail.com
}

\section{Resumo}

A constatação de hábitos de estudo como uma ampla classe de respostas, sugere a investigação de variáveis dependentes e independentes que delas participam ou nelas influem, históricas ou atuais na aquisição e manutenção de comportamentos pró-estudo. Desse modo, muitas variáveis podem ser estudadas como participantes desses processos comportamentais. A presente pesquisa fez um levantamento de contribuições da psicologia comportamental na investigação e intervenção sobre hábitos de estudo, identificando variáveis associadas à aquisição e manutenção desses comportamentos e descrevendo contingências associadas ao contexto universitário. Este trabalho descritivo foi desenvolvido a partir do delineamento de fontes bibliográficas, uma pesquisa pura com natureza de dados qualitativos. A identificação de hábitos de estudo possibilita intervenções, programas psicológicos de atuação profissional, ampliando discussões e inovações constantes com utilidade em gerar tecnologias e avanços científicos. A partir desse levantamento bibliográfico, ampliou-se o olhar sobre intervenções em consonância com a compreensão teórica da psicologia, com base nas relações advindas sobre hábitos de estudo e Análise do Comportamento.

Palavras-chave: Hábitos de estudo; Comportamentos pró-estudo; Contexto universitário; Análise do Comportamento.

\section{Abstract}

The finding study habits as a broad class of responses suggests the investigation of dependent and independent variables that take part in them or influence them, historical or current in the acquisition and maintenance of pro-study behaviors. Thus, many variables can be studied as participants of these behavioral processes. 
Through research it was made a survey of contributions of behavioral psychology in research and intervention on study habits, identifying variables associated with the acquisition and maintenance of these behaviors and describing contingencies associated with the university context. This descriptive study was developed from the design of bibliographic sources, a pure research nature of qualitative data. Identification of study habits allowed possible interventions, psychological programs of professional experience, broadening discussions and constant innovation with utility in generating scientific and technology advances. From this literature review, widens the look on interventions in line with the theoretical understanding of psychology, based on relationships that come about study habits and behavior analysis.

Keywords: study habits; pro-study behaviors; university context; Analysis of Behavior.

\section{Introdução}

Esse artigo se propôs a fazer um levantamento de contribuições da psicologia comportamental, investigando o desenvolvimento e manutenção de hábitos de estudo em contexto universitário.

A partir da revisão proposta têm-se um estudo sobre o que está sendo descrito e o que faz parte da produção nacional científica sobre o assunto, apresentando como objetivo "descrever contribuições da psicologia comportamental sobre hábitos de estudo em contexto universitário". Para essa descrição, apresentou-se contribuições teórico-metodológicas e objetivou-se caracterizar quais as práticas possíveis diante da análise do comportamento sobre hábitos de estudo, variáveis associadas à sua ocorrência, manutenção e possíveis intervenções psicológicas relacionados.

Esse é um tema cujo objeto, hábitos de estudo, corresponde a uma ampla classe composta de muitas variáveis, cuja noção de hábito é compatível ao efeito conjunto dessas no comportamento de estudar e sua frequência, podendo ser agrupadas didaticamente em dimensões: biológica, comportamental (motoras e cognitivas), cultural e ambiental (ambiente físico).

A constatação de hábitos de estudo como uma ampla classe de respostas que o estudante emite em sua interação com o meio que de alguma forma interfere em seu desempenho acadêmico, sugere também a investigação de variáveis dependentes e independentes que delas participam ou nelas influem, históricas ou 
atuais, na aquisição e manutenção de hábitos pró-estudo, bem como nas mudanças observadas a partir de hábitos e comportamentos em algum nível pré-existentes.

Como bem coloca Costa (2002), essa construção social feita pelo indivíduo aumenta a possibilidade de repetição de determinados comportamentos através do fortalecimento ou enfraquecimento de determinadas respostas comportamentais e pode produzir novos comportamentos e novas práticas culturais, sendo assim, reforçamentos devem ser direcionados para produzir consequências nos padrões de respostas e sucessivamente nos padrões sociais e culturais inclusive alterando modelos educacionais.

Muitas variáveis podem ser estudadas como participantes desses processos comportamentais e respostas observadas. Veja que, quando se trata do reconhecimento de que variáveis biológicas, comportamentais e culturais e ambientais estão presentes nesses processos que levam aos hábitos de estudo, pode-se desde já reconhecer que diversas ciências podem estar se voltando para estudar algum aspecto que influa em seu padrão e na forma de aprendizagem.

Observa-se um crescente número de produções científicas que relacionam o rendimento acadêmico com o aprender a estudar. Essas discussões sobre hábitos de estudo se evidenciam nas produções científicas ao longo dos anos e o aumento desses dados corroboram para pensar o hábito de estudo e seus desdobramentos enquanto objeto de estudo sistêmico pela comunidade científica.

A análise do comportamento diante de princípios básicos da aprendizagem é fundamental na compreensão do comportamento humano e sua relação com processo de aprendizagem (HENKLAIN; CARMO, 2013). Esse modelo descreve uma investigação ampla e sistêmica no sentido de considerar os fatores genéticos, operacionais e culturais que envolvem os problemas apresentados pelo indivíduo, para assim buscar hipóteses, a testagem dessas na perspectiva de prever comportamentos futuros e auxiliar na manutenção de atividades desenvolvidas cotidianamente (LETTNER, 1998).

Essas limitações (biológicas, psicológicas, sociais e culturais) que envolvem o estudante, o desconforto diante de resultados insatisfatórios e essas falhas na aquisição de respostas pretendidas, podem associar-se a uma prática ou hábito e consequentemente, dificuldades para atender às demandas do mercado profissional. Um dos agravantes desse processo são os desdobramentos nos cenários 
educacionais, no desenvolvimento de habilidades individuais e na saúde psicológica dos sujeitos envolvidos.

A metodologia que compôs o artigo teve como finalidade a pesquisa pura com natureza de dados qualitativos, na perspectiva de compreender a relação dos fenômenos que envolvem hábitos de estudo e esclarecer a importância do tema em estudo relacionando o indivíduo e questões socioculturais.

O método empregado na pesquisa envolveu a revisão de literatura, considerando fontes bibliográficas advindas de buscas ativas sobre textos que discorrem sobre hábitos de estudo e comportamentos pró-estudo em diferentes áreas de atuação.

Configura-se enquanto uma pesquisa descritiva, uma vez que propôs enquanto objetivo "a descrição das características de determinado fenômeno e então, o estabelecimento de relações entre variáveis" (GIL, 2002. p.42). Através dessa proposta de construção científica entende-se a finalidade de estabelecer relações entre as variáveis associadas à aquisição e manutenção de comportamentos pró-estudo e dimensionar as dificuldades associadas ao seu exercício diante da variabilidade de objetos de estudo relacionado a hábitos de estudo em contexto universitário.

Esta pesquisa foi desenvolvida a partir do delineamento de fontes bibliográficas que possibilitaram "ao investigador a cobertura de uma gama de fenômenos muito mais ampla do que aquela que poderia pesquisar diretamente" (GIL, 2002. p. 44), sendo possível a articulação de conceitos e relações pertinentes à compreensão de hábitos de estudos e programas psicológicos relacionados ao contexto universitário.

A busca dos materiais para compor a pesquisa se iniciaram em janeiro /2019 e os critérios de inclusão inicial foram trabalhos que faziam referência a definição de hábitos de estudo, comportamento de estudar e variáveis relacionadas. Sendo utilizadas palavras-chave como: comportamento de estudar, hábitos de estudo, comportamento pró-estudo, seguindo o viés da análise do comportamento, visando uma melhor elucidação do tema proposto, relacionando esses achados com a aquisição de comportamentos desejáveis ao contexto universitário. Utilizando para tal livros da análise do comportamento e para a busca ativa de artigos e teses foram utilizadas palavras-chave como: hábitos de estudo em contexto universitário, autocontrole comportamental e rendimento universitário. 
Diante da proposta de trazer as contribuições da análise do comportamento para aquisição de hábitos de estudo em contexto universitário, delineou-se em um terceiro momento materiais que relacionavam campos de atuação da psicologia e programas de intervenção relacionados a hábitos de estudo. Sendo utilizadas para tal, as palavras chave: psicologia em contexto universitário, análise do comportamento para hábitos de estudo.

Os critérios estabelecidos para exclusão de materiais foram obras que faziam referência exclusivamente direcionada a professores e instituições de ensino sem mencionar comportamentos dos aprendentes. A caracterização sobre hábitos de estudo foi elaborada por meio de autores da análise do comportamento que possibilitaram uma ampliação do conceito de hábitos relacionando-os a práticas de estudo, além da articulação com variáveis relacionadas à aquisição e manutenção desses comportamentos no âmbito universitário, evidenciando como base os preceitos da análise do comportamento sobre aprendizagem e autocontrole comportamental.

Para tal, esta pesquisa fez-se, sobretudo, de materiais já publicados. Estes materiais bibliográficos incluíram artigos de periódicos científicos, livros e dissertações que foram selecionadas por meio das plataformas digitais em sites de pesquisa acadêmica, como Scielo [Scientific Eletronic Library], BVS-PSI [Biblioteca Virtual em Saúde - Psicologia Brasil] obtidos diretamente na WEB "World Wide Web", pelo programa de busca Google e por meio de publicações impressas sobre o tema.

De posse das obras previamente selecionadas por meio de leitura informativa, foi possível delimitar o material bibliográfico de acordo com os objetivos propostos para elaboração do trabalho científico. Posteriormente, foi realizado a leitura seletiva sobre hábitos de estudo em contexto universitário a fim de articular com possíveis campos de atuação.

A construção de fichas e resenhas foram procedimentos adotados em todas as obras utilizadas na pesquisa, a fim de obter as articulações necessárias entre a construção histórica de conceitos da análise do comportamento que se articulam com as perspectivas acadêmicas $e$ as variáveis associadas à aquisição e manutenção de hábitos pró-estudo. A elaboração de registros digitais em planilhas eletrônicas auxiliou a produção escrita científica e possibilitaram a articulação de autores, obras selecionadas, temáticas correlacionadas ao tema, níveis de 
prioridade das informações e tempo disponibilizado para a execução das tarefas, através de tabelas comparativas de comportamentos elencados pelos autores.

Por meio de todo processo de armazenamento das informações e eliminação de alguns materiais coletados através do controle das informações obtidas, foi possível a articulação sistêmica de relações entre variáveis associadas à aquisição, ocorrência e manutenção de comportamentos pró-estudo no âmbito universitário ampliaram a compreensão sobre o fenômeno estudado.

\section{Classe de comportamentos pró-estudo}

O estudo relacionado aos hábitos pró-estudo dirigirem-se para pesquisas diversas a depender dos aspectos considerados como relevantes para o fenômeno em destaque (ler, escrever, fatores concorrentes, organização pessoal e temporal do indivíduo). Como ressalta Flores (2017) envolve comportamentos e efeitos ambientais em uma perspectiva triádica (bio, psico, cultural/social). Isso adverte, portanto, para níveis de especificidade de análise que podem derivar desses estudos diversos, relatando como certos fatores influenciam ou determinam padrões comportamentais relacionados aos hábitos de estudo e estão relacionadas ao processo de autonomia e controle que o sujeito desenvolve nas habilidades de estudo.

Ao decorrer das produções científicas e diante de uma perspectiva múltipla de fatores determinantes para aquisição de hábitos de estudo, envolvendo o indivíduo e os diferentes ambientes inseridos, como aponta Colombini (2018), hábitos de estudo perpassa a aquisição de respostas adequadas à prática escolar e de estudos apenas para fins acadêmicos, e se relaciona ao processo de desenvolvimento de repertórios comportamentais voltados para o estudo, alcance de metas pessoais, desenvolvimento de autocontrole na ação humana, organização do tempo e estudo e principalmente a possibilidade de aquisição de respostas que sejam funcionais para a resolução de problemas cotidianos, voltados para o estudo e a eliminação de comportamentos que possam dificultar o desenvolvimento de habilidades para estudar.

Hábitos de estudo no plural se refere aos comportamentos pertencentes a uma ampla classe de respostas pró-estudo, uma unidade de análise, onde hábitos seriam comportamentos de estudar diversos que levem a um desempenho 
acadêmico pretendido, e mensurado por algum critério que defina esse desempenho e seu grau (COLOMBINI, 2018). O comportamento de estudar se relaciona a ocorrência dos comportamentos que compõem a classe de resposta 'estudar', envolvem ações do indivíduo voltadas para o estudo em seus diferentes níveis (PERGHER et al., 2012).

Essa é uma definição ampla da aquisição de hábitos de estudo enquanto uma ação complementar das atividades acadêmicas. Como menciona Guilhardi (2002) envolve métodos, estratégias de treino contextualizadas e uma ampla classe de respostas que aumentam a probabilidade futura de emissão de comportamentos pró-estudo, investigando fatores associadas à ação propriamente dita em um processo contínuo em que comportamentos de estudar são instalados em um processo de modelagem e treino que pode levar ao desenvolvimento de hábitos de estudo.

Nesse processo de desenvolvimento de hábitos de estudo, se faz relevante relacionar a todo processo de aquisição e manutenção o contexto que o indivíduo está inserido e as variáveis que compõe esse espaço, o comportamento e as consequências advindas da classe de comportamentos 'estudar', dimensionando o repertório comportamental do indivíduo para um estudo funcional que possibilita resultados positivos e satisfatórios (ZAMIGNANI; KOVAC; VERMES, 2007).

Atribuir aos hábitos de estudo característica de ação que pode ser aprendida e aprimorada na aplicação em diferentes contextos é o fator que permite pensar melhorias na ação humana e sua evolução, como aponta Carelli e Santos (1998) são estratégias passíveis de produzir a generalização desses comportamentos e determinante para a diminuição de condições aversivas. É um esforço integral e contínuo que requer a utilização de planejamentos de rotinas de estudo, o estabelecimento de metas apropriadas aos objetivos do sujeito, analisando as condições pessoais e temporais que 0 indivíduo dispõe para a emissão desses comportamentos.

Estanqueiro (2008) ressalta que o estudo de como estudar deve envolver métodos adequados para a generalização de respostas satisfatórias a diferentes ambientes de estudo, fundamentando a necessidade de 'aprender a estudar' enquanto estratégia de treino e utilização de técnicas que possibilitem ao indivíduo alcançar hábitos de estudo satisfatório aos objetivos propostos e ao contexto que se deseja responder satisfatoriamente. 


\subsection{Hábitos de estudo em contexto universitário}

O universo acadêmico envolve muitas expectativas do acadêmico e geram transformações nos diferentes âmbitos do indivíduo, na esfera pessoal, familiar, social, e como tal, consiste em um período de transição e um processo de ajustamento. Como apontam Glaser e Bardagi (2011), é nesse ambiente que se evidenciam as áreas de interesse profissional, os objetivos que se almeja alcançar e possíveis estratégias futuras de ações coerentes com o saber profissional. O que exige do profissional em formação o desenvolvimento de habilidades e a aquisição de comportamentos mais assertivos a inovadores modos de traçar metas.

Freire (2009) ressalta que para aprendizagem não basta conhecer os conteúdos formais e curriculares. A aprendizagem vai se relacionar com a aquisição de respostas de autorregulação e autonomia no processo de aprender. $O$ indivíduo deve se colocar disponível para o novo, para as ideias inusitadas, o que exige criatividade, criticidade e domínio para agir e gerir informações.

Segundo Basso et al. (2013), a inserção em âmbito universitário envolve uma série de mudanças que exigem do indivíduo habilidades para interagir em novos meios sociais, habilidades e classes comportamentais que possam promover resoluções cotidianas mais eficazes às exigências do ambiente universitário. Segundo Glaser e Bardagi (2011) a tomada de decisões, autonomia, resolução de problemas cotidianos, domínio sobre o processo de estudar, relacionamento interpessoal dentre tantas outras classes de comportamentos representam às exigências de um contexto que cobra do indivíduo maior competência social e melhores habilidades para lidar com as diversificadas situações que o ensino superior podem lhe apresentar, já mensurando uma conduta profissional.

Estudar de forma bem-sucedida e desenvolver hábitos de estudo eficiente deve considerar as condições pessoais do aprendente, que seriam as habilidades que o indivíduo dispõe para a execução das tarefas. As condições temporais para o estudo; tempo que se dedica aos objetivos acadêmicos também devem ser mensurados para se trabalhar competências comportamentais que auxiliem em maior responsabilidade na condução da aprendizagem (CARELLI; SANTOS, 1998). Visto assim, a característica do indivíduo enquanto ser social que se constitui a partir 
das interações com este outro, é possível pensar a relevância das competências sociais (CAMACHO; VILA, 2009).

Desse modo, reforçar e desenvolver comportamentos pró-estudo evidenciam repertórios comportamentais positivos. Como apontam Pergher et al. (2012) o reforçamento é critério fundamental para a aquisição desses comportamentos desejados e sua manutenção. Diante de certa possibilidade de aversão ao comportamento de estudar, é relevante conciliar essa prática com eventos concorrentes agradáveis e prazerosos, enquanto estratégia funcional para a manutenção do comportamento.

Segundo Flores (2017) é possível perceber que as variáveis associadas à ocorrência de comportamentos pró-estudo se direciona a condições pessoais e condições temporais do estudo que se dividem em diversas classes de comportamentos possíveis sobre hábitos de estudo.

Colombini (2018) ressalta o elo de contingências que estão envolvidas na aprendizagem de classes comportamentais para hábitos de estudo. Sendo composto por estímulos antecedentes satisfatórios, ambiente para estudo adequado, programação e objetivos do estudo. Esse elo propicia respostas positivas nas atividades pró-estudo executadas. Com isso, o conhecimento pode ser contextualizado, compreendido e adquire funcionalidade cotidiana que pode ser inserida nos diferentes ambientes do indivíduo.

Sobre essas condições favoráveis ao treinamento dos hábitos de estudo em contexto universitário, é relevante salientar a leitura enquanto condição pessoal necessária. Aperfeiçoar os métodos de leitura favorece o domínio de conteúdo. Saber como desenvolver comportamentos de ler, interpretar e argumentar favorecem a criatividade na busca de conhecimento (CARELLI; SANTOS, 1998). Como foram mencionados por Oliveira et al. (2016) através da leitura acadêmica se inicia um processo de aquisição e manutenção de respostas que transformam o conhecimento adquirido em comportamentos e condutas aplicadas ao cotidiano.

Nesse sentido, Guilhardi (2002) ressalta que fatores relacionados à história individual de cada sujeito e o prévio repertório comportamental desse indivíduo, devem ser quesito norteador dadas às contingências de ensino que integram a relação do sujeito. Se faz necessário analisar o histórico de ações do indivíduo nos diferentes contextos, avaliar as variáveis de contexto familiar em relação às exigências universitárias e como tal, exige a necessidade de considerar o nível de 
interesse gradual que o indivíduo dispõe durante esse processo de formação, para assim, analisar variáveis específicas a esse ambiente dimensionando qual o período o acadêmico cursa, quais as perspectivas metodológicas da formação escolhida, quais os tipos de leitura, de atividades propostas durante essa formação, quais as habilidades exigidas diante da formação teórica e relacionar ao repertório de ações que esse sujeito dispõe.

Assim, pensar o baixo rendimento acadêmico e as dificuldades relacionadas à aquisição e manutenção de comportamentos pró-estudo em contexto universitário, devem ser consideradas quais comportamentos compõem essas dificuldades e identificar possíveis variáveis relacionadas, considerando os programas de ensino e as contingências reforçadoras, na perspectiva de desenvolver habilidades e competências comportamentais (AGASSI, 2013)

Nessa mesma direção supõe Flores (2017), quanto menos contingências relacionadas ao desenvolvimento de comportamentos pró-estudo, maior pode ser a dificuldade em acompanhar as exigências do contexto. Consequentemente surgem processos comportamentais limitantes e as habilidades que o indivíduo dispõe para atender a realidade desse novo cenário se tornam insuficientes para atingir objetivos maiores.

Guilhardi (2002) ressalta que o sono, o cansaço, as rotinas de trabalho, podem ser quesitos que corroboram para esse afastamento de práticas de estudo satisfatórias pois essas exigem planejamento prévio, organização do tempo e compromisso contínuo com objetivos propostos a longo prazo. Como apontam Pergher et al. (2012) envolvendo aspectos de iluminação, estímulos visuais e auditivos do local, organização do ambiente, com horários e materiais para estudo desorganizado e não atraentes para a execução das tarefas, esses fatores dificultam a aquisição de hábitos satisfatórios para o processo de aprendizagem eficaz e a motivação para se dedicar às tarefas necessárias.

Pensar o contexto universitário de formação profissional está diretamente relacionado à capacidade do indivíduo de lidar com situações extremas, lidar com frustrações, autoridade, imprevistos e assim, envolve sua capacidade adaptativa. $\mathrm{O}$ investimento na vida acadêmica é fundamental para se pensar o desenvolvimento de habilidades positivas para esse contexto (SOARES, 2015).

Para além de classes comportamentais específicas como leitura, planejamento pessoal e temporal, à aquisição e manutenção de hábitos de estudo 
envolve também uma outra classe de comportamentos, a capacidade adaptativa do indivíduo. Como bem coloca Glaser e Bardagi (2011) o âmbito universitário se direciona a comportamentos mais assertivos do indivíduo que the permite um bom desempenho social, enquanto uma habilidade facilitadora de assimilação com essa nova realidade de contexto universitário.

\section{O fazer da Psicologia diante de hábitos de estudo em contexto universitário}

Apesar da psicologia focar sua atuação na ótica psicológica, pensar o cenário de atuação profissional do psicólogo relacionado à aquisição e manutenção de hábitos de estudo em contexto universitário, não é um percurso linear e isento de transformações, levando em consideração suas diferentes áreas de conhecimento e os novos movimentos multiprofissionais que influenciam o fazer psicológico, envolvendo as diferentes áreas da psicologia, a pedagogia, as tecnologias digitais de informação e comunicação, que são âmbitos que interferem diretamente no modo como esse indivíduo aprende e portanto, nas possíveis dificuldades associadas.

Os treinamentos dessas competências sociais poderiam sinalizar para um melhor aproveitamento do futuro profissional, promovendo um enfrentamento maior das decisões de carreira e uma conduta mais ativa sobre as situações que se apresentam nas variáveis universitárias (GLASER; BARDAGI, 2011).

De acordo com Henklain e Carmo (2013) pensar a análise do comportamento e suas contribuições para a educação direcionam a ideia de previsão do comportamento humano, onde seria possível dimensionar práticas de ensino que possam aumentar a probabilidade de que aprendentes possam adquirir hábitos com resultados satisfatórios e condizentes ao contexto educacional.

Segundo Souza (2009) o psicólogo tem uma ampla área de atuação no qual pode exercer diferentes papéis com o objetivo de promover o bem-estar do indivíduo mediante as situações que se apresentam. Em contexto de atuação educacional, envolvendo a aprendizagem do indivíduo, é relevante compreender a função a ser desenvolvida por profissionais que desejam trabalhar a aquisição e manutenção em prol de hábitos de estudo mais assertivos.

Como apontam Kienen et al. (2017) em cenário universitário, por meio da inserção da tecnologia e das novas formas de programação de ensino seria possível construir condições que possam ampliar a possibilidade do aprendente transformar o 
conhecimento adquirido em comportamentos altamente eficazes ao meio, de modo que a tecnologia possa servir a melhorias na ação humana.

Agassi (2013) descreve 'programar condições de ensino' enquanto o fazer de profissionais envolvidos na educação e cita a psicologia enquanto possibilidade de atuação em que o profissional seja um facilitador responsável por auxiliar o desenvolvimento de repertórios comportamentais voltados para a aprendizagem do indivíduo, evidenciando a clareza que esse profissional deve ter em relação aos processos múltiplos envolvidos no ensinar e aprender dos indivíduos.

Kienen et al. (2017) propõem para a tarefa do psicólogo em desenvolver estratégias de estudo, a organização do ambiente de estudo, realizar o planejamento de tarefas e análise do tempo disponibilizado, a observação desses aspectos poderia auxiliar na articulação dos saberes e campos relacionais com a resolução de problemas cotidianos.

Por meio de metodologias de acompanhamento terapêutico e planejamentos sistêmicos, seria possível propor uma atuação profissional fundamentada em contingências tecnológicas envolvidas no processo de ensino. O profissional da psicologia viabiliza uma análise das contingências que estão em vigor, fatores determinantes do comportamento, e o planejamento futuro a partir de análise funcional contínua, na perspectiva de auxiliar a manutenção e generalização de comportamentos mais adequados às exigências do mercado atual (FLORES, 2017).

Análise funcional possibilita uma investigação minuciosa das contingências que estão em vigor diante de um comportamento. Como menciona Camacho e Vila (2009) a análise funcional das variáveis antecedentes, consequentes e mantenedoras que favorecem a aquisição de hábitos pró-estudo, possibilitam a identificação de dificuldades associadas e permite a modificação de comportamentos indesejáveis. Pensando assim, de modo relacional, qual a função exercida por esse comportamento para o indivíduo e para a relação com o ambiente.

Desse modo, não existe método de estudo ideal, existem técnicas que se contextualizam às necessidades do contexto e objetivos do indivíduo. Podemos mencionar Hubner e Marinotti (2004) a aquisição e manutenção de hábitos de estudo devidamente instalado no repertório comportamental do indivíduo, quando este emite classes de comportamentos pró-estudo que se repetem diante dos contextos de modo estável e dão origem a uma produção comportamental que possibilita mensurar o processo de aprendizagem do indivíduo. Milan e Peter (2007) 
descrevem etapas a serem orientadas para que o indivíduo melhore a aquisição de conhecimento e aprendizagem nos estudos por meio do autocontrole comportamental.

Milan e Peter (2007) orientam a ensinar os aprendentes manejos de autoobservação e auto-registro para que posteriormente, sirva de parâmetro às avaliações de outras pessoas, inclusive para a intervenção psicológica. O passo seguinte consiste em ensinar o cliente a se auto-instruir e guiar seu próprio comportamento, organizando o ambiente e variáveis para reforçar o comportamento e respostas que se deseja obter. Aos poucos o analista do comportamento deve transferir o controle dos reforçamentos para o próprio cliente, retirando gradativamente as contingências instauradas para testagem do comportamentoalvo. De modo que o indivíduo passe a controlar de maneira eficaz seu comportamento.

Agassi (2013) considerando o comportamento de ler em contexto universitário, uma classe de comportamentos que desenvolvem outras classes comportamentais na aquisição e manutenção de hábitos pró-estudo, descreve um programa de desenvolvimento de condições de ensino em etapas que considerem comportamentos objetivos para o estudo. Utilizando para tal, roteiros com os objetivos de leitura, elaborando perguntas que orientem essa leitura, elencando quais unidades textuais consegue reproduzir com as próprias palavras, para posteriormente escrever as considerações relevantes sobre a leitura e por fim, avaliar como foi o próprio comportamento de ler diante das etapas seguidas.

Essas etapas e procedimentos correspondem a processos que devem ser desenvolvidos através da utilização de programas de intervenção baseados nos preceitos da psicologia, buscando auxiliar o indivíduo em ações funcionais de modo gradativo, na perspectiva de programar unidades de aprendizagem mais específicas e acessíveis ao repertório comportamental que o indivíduo dispõe.

\section{Considerações finais}

Mediante a proposta de descrever as contribuições da psicologia comportamental sobre hábitos de estudo em contexto universitário, foi possível identificar variáveis associadas à aquisição e manutenção de hábitos de estudo e descrever as contingências associadas à essa aquisição, manutenção e as dificuldades de seu exercício, e assim, compreender possíveis 
programas/intervenções da Psicologia sobre esses hábitos, conforme variáveis relacionadas.

A prática educacional se serve na psicologia das muitas especialidades existentes e todas elas podem ser úteis a depender do tipo de intervenção requerida. Assim, os estudos na área de educação e análise do comportamento seguem o mesmo raciocínio, exatamente por integrar o propósito em seu auxílio ao campo da educação, entender os fenômenos de interesse e disponibilizar meios para promoção de ações nessa área e solução de problemas existentes nesses campos.

Essa perspectiva de interação entre fatores que determinam uma ação, possibilitou pensar a mudança de ações (comportamento) e ampliou o olhar para possibilidades de aprendizagem, habilidades e competências que o sujeito possa desenvolver diante de seu repertório genético, histórico e sociocultural, mediante os preceitos do processo de aprendizagem e modificação do comportamento que são evidenciados nas obras da análise do comportamento.

As investigações relacionadas ao ensino de comportamentos e ao processo de aprendizagem humana constituem-se um objeto de estudo da análise do comportamento e uma possibilidade de intervenção profissional. Essas são contribuições da análise do comportamento para a educação, possibilitar o estudo da ação humana partindo de um novo olhar sobre o sujeito que se comporta, dimensionando esse indivíduo enquanto sujeito ativo transformador das realidades do qual ele participa, sendo uma construção dialética.

As literaturas analisadas sobre o tema hábitos de estudo evidenciam a conduta do senso-comum em atribuir de modo interno a motivação e responsabilidade ao indivíduo quanto ao 'sucesso' ou 'insucesso' nos estudos, desconsiderando a complexidade da aquisição de hábitos pró-estudo e a variabilidade de fatores que podem determinar os resultados no processo de estudar.

Essa proposta de treinar comportamentos pró-estudo, ainda é uma problemática que incita investigação. Esse fazer psicológico com estratégias funcionais voltadas ao saber educacional ainda se relacionam ao processo de aprendizagem e as variáveis associadas. Essas concepções ainda precisam ser desconstruídas e repensadas no contexto histórico e cultural ao qual dado comportamento está inserido. 
As classes comportamentais voltadas para hábitos de estudo, possibilitaram o direcionamento para o contexto universitário enquanto possibilidade de capacitar futuros profissionais na tomada de decisão e autonomia em trazer resoluções constantes para problemas cotidianos que o indivíduo vivencia diante de processos deficitários na educação superior. Ampliando a possibilidade de repensar o fazer psicológico e produzir intervenções qualificadas às demandas e objetivos do indivíduo.

Diferentes campos de atuação podem se beneficiar desse novo olhar sobre o comportamento humano, não se tratando apenas de técnicas previstas para cada ação, mas sim de um novo modo de intervir às demandas que o sujeito evidencia nos diferentes âmbitos profissionais. É um olhar peculiar da Psicologia sobre o sujeito-organismo que se comporta e ativamente pode modificá-lo, desenvolvendo as relações e interações que vai selecionando no ambiente e produzindo um repertório de ações bem-sucedidas.

\section{Referências}

AGASSI, I. Avaliação da eficácia de um programa de condições de ensino para desenvolver ou aperfeiçoar a cadeia de classes de comportamentos "Ler Textos em Contexto Acadêmico" como parte do repertório de estudantes de cursos de graduação. 2013. 349 p. Dissertação (Mestrado) em Universidade Federal de Santa Catarina, Centro de Filosofia e Ciências Humanas, Florianópolis, 2013. Disponível em: $\leq$ https://repositorio.ufsc.br/handle/123456789/122737>. Acesso em: 10 ago. 2019.

BASSO,C. et al. Organização de tempo e métodos de estudo: oficinas com estudantes universitários. Revista Brasileira de Orientação Profissional, Florianópolis v.14, n. 2, jun-dez., 2013. Disponível em $<$ http://pepsic.bvsalud.org/pdf/rbop/v14n2/12.pdf> Acesso em 09 de ago./2019

CAMACHO, A.; VILA, E. M. Levantamento de variáveis antecedentes e consequentes que favoreceram a aprendizagem e a manutenção de dificuldades interpessoais em universitários. Semina: Ciências Biológicas e da Saúde, Londrina, v. 30, n. 1, jan./jun, 2009. Disponível em <http://www.uel.br/revistas/uel/index.php/seminabio/article/view/2900/2458> Acesso em 10 de ago./2019

CARELLI. M. J. G.; SANTOS. A.A.A. Condições temporais e pessoais de Estudo em Universitários. Psicologia, Escola, Educação. Universidade São Francisco, Campinas, v.2, n.3, 1998. Disponível em $<$ http://www.scielo.br/pdf/pee/v2n3/v2n3a06> Acesso dia 19 de Jan/2019 
COLOMBINI, F. A. Análise da Produção Científica Brasileira sobre o Estudar. 2018. 182 p. Dissertação (Mestrado em Educação: Psicologia da Educação), em Pontifícia Universidade Católica de São Paulo, São Paulo, 2018. Disponível em $<$ https://tede2.pucsp.br/handle/handle/21118> Acesso em 18 de Jan./2019.

COSTA, N. Terapia analítico-comportamental: Dos fundamentos filosóficos à relação com o modelo cognitivista, Santo André, SP. ESETec Editores Associados, 2002.

ESTANQUEIRO, A. Aprender a estudar: um guia para o sucesso na escola. 13.ed. Campinas: Texto editora, 2008

FLORES, E. P. Análise do Comportamento: Contribuições para a psicologia escolar. Revista Brasileira de Terapia Comportamental e Cognição, Brasília, v. 2, n.1, 2017. Disponível em <https://doi.org/10.31505/rbtcc.v19i1.955> Acesso em 19 de mar/2019.

FREIRE, L.G.L. Auto-regulação da aprendizagem: Ciências \& Cognição, Lisboa, v.14, n.2, 276-286, 2009. Disponível em < http://www.cienciasecognicao.org/pdf/v14 2/m318358.pdf> Acesso em 09 de ago./2019

GIL, A. Como elaborar um projeto de pesquisa. 4.ed. São Paulo: Atlas, 2002

GLASER, S. L.; BARDAGI, M.P. Habilidades sociais, auto-eficácia e decisão de carreira em universitários em no final de curso: Boletim Academia Paulista de Psicologia, São Paulo, v. 80, n.1, 2011. Disponível em $<$ https://www.lume.ufrgs.br/handle/10183/31819 > Acesso em 09 de ago./2019

GUILHARDI, H. J. et al. Sobre Comportamento e Cognição: contribuições para a construção da teoria do comportamento. v. 9, Santo André: ESETec Editores associados, 2002

HENKLAIN, M. H. O.; CARMO, J. S. Contribuições da Análise do Comportamento à educação: Um convite ao diálogo. Cadernos de Pesquisa, São Carlos, v.43, n.149, maio-ago, 2013 Disponível em < http://www.scielo.br/pdf/cp/v43n149/16.pdf> Acesso em 09 de ago./2019

HUBNER, M. M. C.; MARINOTTI, M. Análise do comportamento para a Educação: Contribuições Recentes, aprender a estudar. v.2, Santo André, ESETec Editores associados, 2004

KIENEN, N. et al. Comportamentos pré-requisitos do "Estudar textos em contexto acadêmico". Rev. CES Psicologia, Londrina, v.10, n. 2, 2017. Disponível em $<$ http://www.scielo.org.co/pdf/cesp/v10n2/2011-3080-cesp-10-02-00028.pdf> Acesso 09 de ago./2019

LETTNER, H. W. Avaliação comportamental: Psicoterapia comportamental e cognitiva de transtornos psiquiátricos, Campinas, 1998. 
MILAN A. M.; PETER. Z.M. Manual de Técnicas de Terapia e Modificação Comportamental: A Generalização e a Manutenção dos Efeitos do Tratamento. Santos. 1.ed., São Paulo, 2007

PERGHER. N. K. et al. Desenvolvimento de Hábitos de Estudo, Pró-estudo. In. BORGES, F. A. Clínica analítico-comportamental: aspectos teóricos e práticos. Porto Alegre: Artmed, 2012

SOARES, A.B. Habilidades Sociais e Vivência Acadêmica de Estudantes Universitários: Interação em Psicologia, Curitiba, v. 19, n. 2, maio/ago. 2015. Disponível em <https://revistas.ufpr.br/psicologia/article/view/31663> Acesso em 10 de ago./2019

SOUZA, M. P. R. Psicologia escolar e educacional em busca de novas perspectivas. Revista Semestral da Associação Brasileira de Psicologia Escolar e Educacional (ABRAPEE). São Paulo, v. 13, n. 1, jan-jun, 2009. Disponível em: $<$ http://pepsic.bvsalud.org/pdf/pee/v13n1/v13n1a21.pdf > Acesso em 09 de ago./2019

ZAMIGNANI, D. R.; KOVAC, R.; VERMES, J. S. A Clínica de Portas Abertas: experiências e fundamentação do acompanhamento terapêutico da prática clínica em ambiente extraconsultório. São Paulo: Paradigma, 2007 